Zeitschriftenartikel:

Begutachtet

Redaktion und Begutachtung:

Nele Heise (iD)

Digital Media \& Communication

Researcher Hamburg

Nils Zurawski (iD

Universität Hamburg

Erhalten: 01. Dezember 2019

Akzeptiert: 29. September 2020

Publiziert: 15. Dezember 2020

Lizenz:

(c) Jürgen Karla

Dieses Werk steht unter einer Lizenz

Creative-Commons-Namensnennung 4.0

(CC-BY 4.0) International

(C) (i)

Datenverfügbarkeit:

Alle relevanten Daten befinden sich innerhalb der Veröffentlichung.

Interessenskonfliktstatement:

Die Autor:innen erklären, dass ihre Forschung ohne kommerzielle oder finanzielle Beziehungen durchgeführt wurde, die als potentielle

Interessenskonflikte ausgelegt werden können.

\section{Empfohlene Zitierung:}

Karla, J. (2020). Kaffeepause-Podcast: Erfahrungen mit der Produktion und dem Einsatz von Podcasts für die Wissenschaftskommunikation in der Lehre.kommunikation@gesellschaft, 21(2). https://doi.org/10.15460/kommges. 2020.21.2.633

\section{Kaffeepause-Podcast}

\section{Erfahrungen mit der Produktion und dem Einsatz von Podcasts für die} Wissenschaftskommunikation in der Lehre

\author{
Jürgen Karla a* iD \\ ${ }^{a}$ Hochschule Niederrhein, Mönchengladbach \\ * Korrespondenz: juergen.karla@hs-niederrhein.de
}

\begin{abstract}
An der Hochschule Niederrhein werden Podcasts für die Wissenschaftskommunikation eingesetzt. Im vorliegenden Beitrag werden die Erfahrungen mit der Produktion von Podcasts in der Lehre durch Studierende dargestellt. Die Strukturierung erfolgt dabei anhand der Prozessschritte 1. Pre-Produktion - Konzeption, 2. PreProduktion - Redaktionsplanung und Briefing, 3. Produktion - Aufnahmetechnik und Aufnahmeprozess, 4. Post-Produktion - Schnitt und Audionachbearbeitung, 5. Publikation. Die gesammelten Erfahrungen werden dargestellt und die Weiterentwicklung des Projektes erläutert.

Schlagworte: Podcast, Wissenschaftskommunikation, Kaffeepause, Lehre, Studierende, Produktion, Evaluation
\end{abstract}




\section{Einleitung}

An der Hochschule Niederrhein werden Podcast-Episoden für die Wissenschaftskommunikation seit dem Sommersemester 2019 eingesetzt. Die Konzeption, Pre-Produktion, Produktion sowie Post-Produktion werden dabei durch Studierende des Studienganges Wirtschaftsinformatik als Teil einer Prüfungsleistung im Modul „Social Media“ unter Anleitung erbracht. Dabei wird den Studierenden die Möglichkeit gegeben, einen Podcast als ein digitales Produkt selbständig zu entwickeln. Den Studierenden soll damit die Möglichkeit zur Erweiterung ihres Kompetenzportfolios gegeben werden.

Inhaltlich wendet sich der Podcast mit den Episoden der ersten Staffel an drei Zielgruppen: a) Schüler:innen soll ein Einblick in den Studiengang Wirtschaftsinformatik ermöglicht werden, b) aktuellen Studierenden sollen Informationen zur Erstellung von Abschlussarbeiten gegeben werden und c) Unternehmen sollen Informationen zu Forschungsthemen an der Hochschule Niederrhein und über den Studiengang Wirtschaftsinformatik B.Sc. zur Verfügung gestellt werden.

Nach Abschluss der ersten Staffel ist eine Evaluation des Podcast-Projekts erfolgt, aus deren Ergebnissen sich eine Überarbeitung des ursprünglichen Konzeptes ergeben hat, welche im Rahmen der Produktion der zweiten Staffel durch die Studierenden des nächsten Jahrgangs umgesetzt wurde.

Die gesammelten Erfahrungen aus der bisherigen Projektumsetzung sollen mit dem vorliegenden Beitrag in strukturierter Form anhand der wesentlichen Projektmeilensteine (1. Pre-Produktion - Konzeption, 2. Pre-Produktion Redaktionsplanung und Briefing, 3. Produktion - Aufnahmetechnik und Aufnahmeprozess, 4. Post-Produktion - Schnitt und Audionachbearbeitung, 5. Publikation) verdeutlicht werden. Erfahrungen aus dem Projekt werden geschildert und die Umsetzung der Evaluationsergebnisse in ein überarbeitetes Konzept dargestellt. Die durchgeführten notwendigen Schritte zur Verstetigung und Überführung aus dem Projektstatus in einen festen Bestandteil der Lehrveranstaltung werden erläutert.

\section{Einordnung}

Hochschulen für Angewandte Wissenschaften streben zur Steigerung der „Employability“ ihrer Absolventen eine intensive Verknüpfung der Wissensvermittlung mit praktischen Anwendungsinhalten an, um so der Forderung von Unternehmen zur Unterstützung in ihrem jeweiligen Wettbewerbsumfeld nachzukommen. Entsprechend sollen Studierende über einen Kompetenzerwerb bestmöglich auf die Herausforderungen in zukünftigen Berufen vorbereitet werden. Hochschulen setzen hier insbesondere auf Lehrinnovationen, um moderne und zukunftsgerichtete Formate für die Lehre zu entwickeln. Im Rahmen einer solchen Lehrinnovation wurde an der Hochschule Niederrhein ein Projekt mit dem Fokus auf eine aktive Wissenschaftskommunikation 
initiiert. Im Rahmen der Lehrveranstaltung „Social Media“ haben Studierende unter Anleitung durch den Dozenten einen Podcast ${ }^{1}$ als eine Form von abonnierbaren Audio-Inhalten konzipiert, produziert und publiziert.

Bereits seit einigen Jahren werden Konzepte wie das Technology-enhanced learning (TEL) wissenschaftlich betrachtet. Gemeint ist damit häufig ein Einsatz von Informations- und Kommunikationstechnik in der Lehre, ohne jedoch eine konkrete Definition dessen zu liefern (Kirkwood/Price 2014). Auch wurde bereits kurz nach der ersten ,Hype-Welle' des Podcastings (2004-2007) über einen Einsatz in der akademischen Lehre geforscht (z.B. McKinney et al. 2009; Ketterl et al. 2006; Kay 2012). Interessant ist hierbei, dass die seinerzeit erkannten Problemfelder (Copley 2007; Kronen/Karla 2007a) des Einsatzes audiovisueller Medien in der Lehre in der heutigen Zeit weitestgehend durch technische und didaktische Lösungen adressiert werden, z.B. technische Hard- und Software-Lösungen, Nutzungsbarrieren, Integration in Content-Management-Systeme.

In dem hier vorgestellten Projekt wurde den Studierenden die Möglichkeit gegeben, einen Podcast als ein digitales Produkt selbständig zu entwickeln. Die Ziele des Konzepts waren mehrschichtig gestaltet: (a) sollte die produzierte Podcast-Reihe als Teil der Prüfungsleistung geltend gemacht werden, (b) somit das Kompetenzportfolio der Studierenden erweitern und (c) über die Publikation der Podcasts Schüler:innen, aktuellen Studierenden und Unternehmen Informationen aus der Hochschule und über den Studiengang Wirtschaftsinformatik B.Sc. zur Verfügung stellen. Inhaltlich wurden in den Podcast-Episoden bislang vorrangig Themen aus Abschlussarbeiten (sowohl Bachelor- als auch Master-Niveau) aufgegriffen und in anschaulicher Weise vermittelt. Dies entspricht den im Konzept für die Lehrinnovation (Karla/Hoffmann 2019) definierten Zielgruppen. Mittels der inhaltlichen Fokussierung können Schüler:innen für Inhalte der Wirtschaftswissenschaften interessiert, aber auch zukünftige Arbeitgeber auf potentielle Mitarbeiter sowie Lehr- und Forschungsthemen des Fachbereichs Wirtschaftswissenschaften an der Hochschule Niederrhein aufmerksam gemacht werden. Ergänzend können Studierende anhand der aufbereiteten Inhalte von den Erfahrungen ihrer Kommiliton:innen profitieren. Mittels der Podcast-Episoden können Forschende der Hochschule ihre Forschungsthemen im Rahmen der Wissenschaftskommunikation platzieren.

In der heutigen Wissenschaft zeigt sich, dass nicht jedes inhaltlich und formal gute Ergebnis einer Abschlussarbeit auch für eine Publikation in einem gedruckten Organ geeignet ist. War die Drucklegung in der Vergangenheit der ,"übliche“ Weg in der Wissenschaftskommunikation, so zeigt sich heute, dass ein Wandel in der Wissenschaft und in der Gesellschaft durchaus neuen Publikationsformen einen Weg ebnen kann. Allgemeine Statistiken belegen, dass die Nutzung von Podcasts derzeit ein stark wachsendes Potential auch für die Wissenschaftskommunikation in sich birgt (Domenichini 2018). Die Erfahrung in der Lehre zeigt, dass Studierende sehr viel Energie und Freude in

1 Website des Kaffeepause-Podcast: http://www.jkarla.de/podcast/ (Zugriff am 19.08.2020). 
die Erarbeitung neuer Erkenntnisse - im Rahmen von Abschlussarbeiten und -projekten - investieren, nur um im Anschluss feststellen zu müssen, dass die Ausarbeitung dann in einem Aktenschrank gelagert wird und nur selten durch z.B. eine Publikation eine Würdigung in größerem Rahmen erfährt. Dabei ist bekannt, dass Studierende die erlernten und erarbeiteten Ergebnisse gerne auch im Kontext der Wissenschaftskommunikation teilen möchten. Diese Problematik sowie die Forderung nach einer stärkeren Verknüpfung der Wissensvermittlung mit praktischen Anwendungsinhalten sollte im Rahmen der Umsetzung der hier vorgestellten Lehrinnovation zu Podcasting in Lehre und Wissenschaftskommunikation adressiert werden. So können Inhalte eines Studiengangs in einer geeigneten Form in die Wissenschaftskommunikation transportiert werden, d.h. Schüler:innen und Lehrer:innen, aber auch Unternehmen einen Zugang zu Inhalten eines Studienganges und ein Verständnis für wissenschaftliche Erkenntnisse ermöglichen (McGarr 2009; Kronen/Karla 2007b).

\section{Ziele der Lehrinnovation}

Die Ziele der hier vorgestellten Lehrinnovation lassen sich dementsprechend mehrschichtig konkretisieren: Studierende wurden in der Nutzung digitaler Medien angeleitet und zur Erstellung dieser befähigt. Die Lehrinnovation sollte dabei einem ganz realen Projektvorhaben entsprechen (Aufgabenstellung, Analyse, Konzeption, Entwurf, Umsetzung, Test, Implementierung). Den Studierenden wurde die Möglichkeit gegeben, ein komplettes Projekt zu durchlaufen und sich in diesem zu erproben. Didaktisch unterstützt wurde das Lernen, indem der Dozent sich aus der Position des frontal Lehrenden zurückzog und den Studierenden in einer Rolle als Coach zur Seite stand. Das fertige Produkt diente anstelle einer Hausarbeit als Prüfungsleistung und hat darüber hinaus das Potential - auch über die Hochschulgrenze hinaus - insbesondere zukünftige Arbeitgeber und zukünftige Studierende zu erreichen. Die Lehrinnovation verfolgt folgende Projektziele:

- Zufriedenheit aller Akteure (Studierende, Dozent, Hochschule, Unternehmen, Schüler:innen)

- Lernerfolg am Ende der Maßnahme (Projektdurchführung, Produktion, Podcast)

- Lerntransfer auf Anwendungssituation

- Kommunikation von Themen aus der Hochschule.

Damit einhergehend wurde mit der Lehrinnovation eine Reihe an Lernzielen angestrebt, die optimalerweise am Ende der Durchführung erreicht werden sollten:

- Die Studierenden können digitale Kommunikationsmedien benennen und diese in ihrer Gesamtheit klassifizieren

- Die Studierenden können ein Konzept (über Projektphasen hinweg) einer Podcast-Produktion entwickeln 
- Die Studierenden können eine Redaktionsplanung für eine PodcastProduktion unter Einbeziehung einer Social-Media-Strategie entwerfen

- Die Studierenden wenden Kenntnisse zu Technik und Prozessen in PreProduktion, Produktion und Post-Produktion an

- Die Studierenden können die Ergebnisse in Form von Podcast-Episoden präsentieren.

\section{Durchführung und Evaluation}

Zur Erreichung der Ziele erfolgte eine Umsetzung anhand der folgend erläuterten Phasen.

\subsection{Pre-Produktion - Konzeption}

Im Rahmen der Umsetzung der hier vorgestellten Lehrinnovation wurden die Rahmenbedingungen für eine digitale Lehre verbessert und eine Investition in Hardware und Software für die Produktion von Audioinhalten getätigt. Das Investment belief sich insgesamt auf einen Betrag von unter 600 Euro für die Anschaffung von

- Zoom H6 Field Recorder (Aufnahmegerät inkl. Aufsteck-Mikrofone)

- Shure SM58 Mikrofone für die Aufnahme der Interviews

- Reaper.fm mit Ultraschall.fm² als Software für die Post-Produktion.

Die genannten Bestandteile zählen zu den de facto-Standards für viele Podcast-Projekte (siehe auch Karla o.J.).

Aus der ersten Kohorte teilnehmender Studierender wurde für die folgenden Durchläufe die Einstellung einer studentischen Hilfskraft vorgenommen, die das Vorhaben - insbesondere aus der technischen und redaktionellen Perspektive (Einweisung in die Technik, Anfertigung der Aufnahmen, Unterstützung bei Pre-Produktion und Post-Produktion, Erstellung von Interview-Konzepten und Rahmenwerken) - unterstützt hat. Entsprechend ist für die Folgedurchläufe ein Budget vorzusehen.

Im Sinne einer didaktischen projektbasierten Methode war außerdem eine Schulung des Dozenten in Coaching-Tools (Methoden, Techniken, Instrumente und Konzepte) sinnvoll. Des Weiteren wurde eine Schulung des Dozenten im Bereich Radiosprechen vorgesehen, mit dem Ziel das dort erlangte Wissen an die Studierenden weitergeben zu können. Für eine initiale Umsetzung war außerdem eine Schaffung zeitlicher Freiräume für den Dozenten notwendig.

Diese Rahmenbedingungen gegeben, konnte während der Durchführung der Lehrveranstaltung die Konzeption des Podcast-Projekts mit den Studierenden angegangen werden. Hierzu wurden in einer initialen Vorstellung zunächst das Ziel des Projekts sowie der grobe Ablauf skizziert. In den

2 https://ultraschall.fm (Zugriff am 19.08.2020). 
Folgeveranstaltungen konnte eine schrittweise Konkretisierung einzelner Teilbereiche adressiert werden. Aus dem Kontext des Moduls „Social Media“ heraus wurde so mit der ersten Kohorte eine Namensrecherche betrieben.

Eine Wettbewerbsanalyse ergab, dass die Bezeichnung ,Kaffeepause' interessanterweise im deutschsprachigen Podcast-Angebot noch unbesetzt war. Auch eine Markenrecherche über das Deutsche Patent- und Markenamt ergab, dass dieser Titel für das Projekt nicht anderweitig belegt war. Um die Ausrichtung der Inhalte auch im Titel zu reflektieren, wurde als Untertitel „Abschlussarbeiten in 15 Minuten“ ergänzt. Die Evaluation der Konzeptionsphase kann der Abb. 1 entnommen werden. ${ }^{3}$

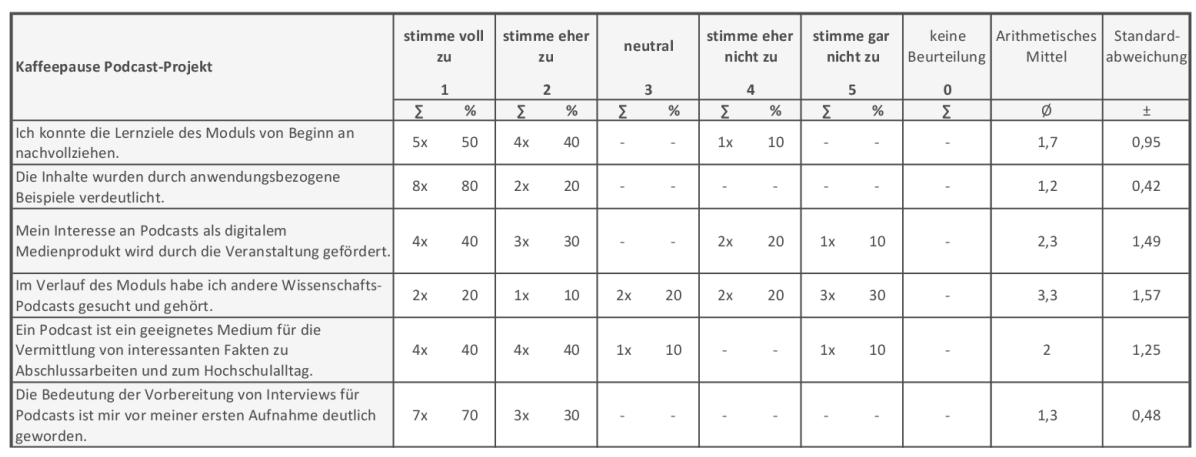

Abbildung 1: Evaluation der Konzeptionsphase $(n=10)$. Quelle: eigene Darstellung

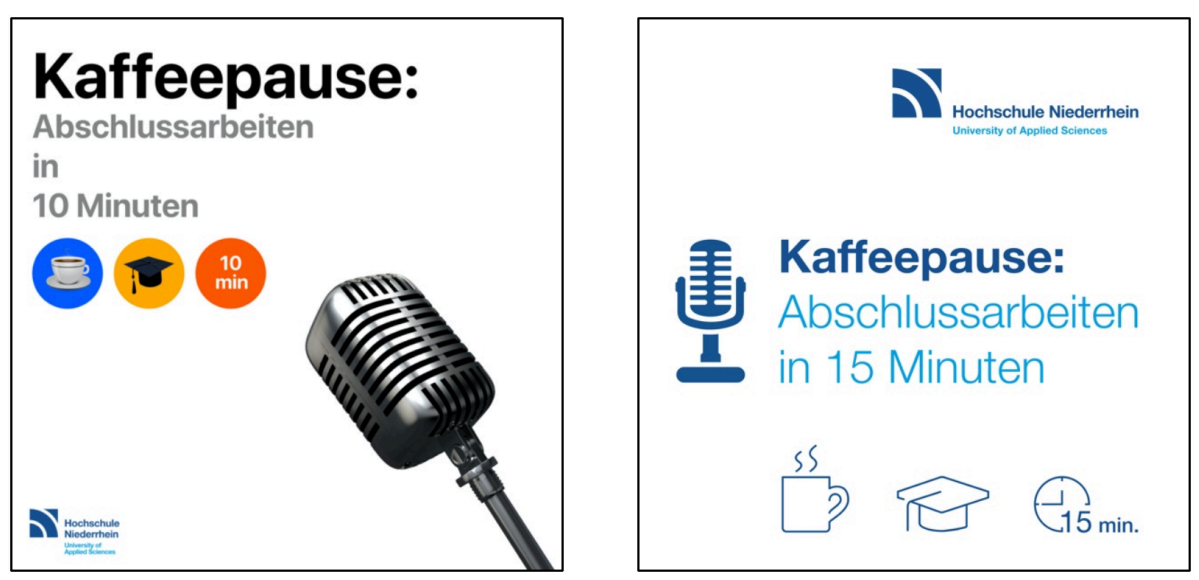

Abbildung 2: Entwicklung des Podcast-Logos. Quelle: eigene Darstellung

Aus der Evaluation lassen sich bereits bedeutsame Aspekte für die Konzeption der Integration einer Podcast-Produktion in eine Lehrveranstaltung ablesen. Lernziele sind für Studierende immer von besonderer Bedeutung, lassen sich hieraus doch die Beurteilungskriterien für Prüfungsleistungen ablesen. Die Einbettung von anwendungsbezogenen Beispielen sorgte für eine hohe Anschaulichkeit. So wurden beispielsweise bestehende Podcast-Angebote betrachtet und grob analysiert. Manchmal hat dies sogar zu einer Steigerung des Interesses an Podcasts geführt.

3 Befragt wurden die zehn Studierenden der ersten Kohorte im Sommersemester 2019 nach Abschluss der Lehrveranstaltung. 
Diese Basis geschaffen, haben die Studierenden im Anschluss ein Logo für das Podcast-Projekt entwickelt. In einem Gruppenprozess erfolgte hier eine schrittweise Verfeinerung. Das abschließende Design des Logos erfolgte unter Beachtung der Corporate Design-Vorgaben der Hochschule Niederrhein (insbesondere Schriften, Farben, Logo-Einbettung) durch das Referat Hochschulkommunikation. Abb. 2 zeigt die Entwicklung vom Entwurf der Studierenden (links) hin zum finalen Logo (rechts). Die für Podcasts üblichen Formatvorgaben wurden dabei beachtet.

\subsection{Pre-Produktion - Redaktionsplanung und Briefing}

Nachdem dieses Fundament für den Podcast gelegt war, erfolgte eine feingranulare Produktions- und Redaktionsplanung. Diese beinhaltete die zeitliche sowie organisatorische Verlaufsplanung. Zu diesem Termin erhielten die Studierenden einen Einblick über den Entwicklungsverlauf eines Projekts (Projektphasenmodelle) sowie die Grob- und Feinkonzeptionierung der Produktion von Podcast-Episoden. Im Anschluss daran mussten die Studierenden in Kleingruppen ein Konzept für ihre Podcast-Episode erstellen. Neben der Terminierung der Aufnahmen der Interviews war ein wesentlicher Bestandteil die Akquise von Studierenden, die sich zu ihren Abschlussarbeiten durch die Kommilitonen interviewen lassen wollten. Hier spielte naturgemäß das persönliche Netzwerk des Dozenten eine vorrangige Rolle. Bei der Akquise war die sorgsame Aufklärung - beispielsweise unter den Aspekten des Datenschutzes - besonders wichtig. Audioaufnahmen beinhalten konkrete personenbezogene Daten und sind entsprechend der jeweiligen geltenden Datenschutzregelungen zu behandeln. Diese Datenschutzaufklärung ist dabei unbedingt zu dokumentieren.

Wesentlichen Anteil für das Gelingen von Interview-Podcasts ist das geeignete Briefing der Interviewpartner. Hierauf wurde entsprechendes Augenmerk gelegt: Die Studierenden erhielten eine Einführung zur Redaktionsplanung sowie über ein Briefing der Gesprächspartner und die Aufbereitung der Inhalte (Stichwort: Personenbezogene Daten im gesprochenen Wort). Im Anschluss daran mussten die Studierenden ein Grobgerüst für einen Redaktionsplan sowie einen Fragenkatalog zum Thema Briefing eigenständig erstellen. Dieser Fragenkatalog wurde anschließend in Kleingruppen sowie im Plenum und mit dem Dozenten besprochen und erneut überarbeitet.

Die jeweiligen Interviewpartner erhielten im Rahmen eines vorbereitenden Gesprächs letztlich den ausgearbeiteten Interviewfragenkatalog.

\subsection{Produktion - Aufnahmetechnik und Aufnahmeprozess}

$\mathrm{Zu}$ diesem Termin erhielten die Studierenden das notwendige Wissen zur Nutzung der einzusetzenden technischen Ausstattung (Aufnahmegerät und Mikrofone). Des Weiteren wurde besonderer Wert auf das Ablegen einer natürlichen Scheu vor dem Mikrofon gelegt. Dazu konnten die Studierenden 
zunächst einmal völlig frei mit der Technik arbeiten und sich an die Nutzung gewöhnen. Sodann hat der Dozent in Kleingruppen die Best Practices im Umgang mit der Aufnahmetechnik erläutert und demonstriert. Ziel hierbei war das Erlernen der sogenannten Mikrofondisziplin, was die Positionierung und Haltung der Mikrofone sowie Atemgeräusche und Schmatzlaute beinhaltete, aber auch die Gestaltung der Aufnahmelokalität (Hall, Umgebungsgeräusche) berücksichtigte. In einer weiteren Erprobung konnten die Studierenden hier erneut in Kleingruppen die verwendete Aufnahmetechnik und den Umgang damit einüben. Im Anschluss erfolgten Probeaufnahmen, die auch im Rahmen der Post-Produktion als Übungsmaterial verwendet wurden.

\begin{tabular}{|c|c|c|c|c|c|c|c|c|c|c|}
\hline \multirow[t]{2}{*}{ Kaffeepause Podcast-Projekt } & $\begin{array}{c}\text { stimme voll } \\
\mathrm{zu} \\
1 \\
\end{array}$ & $\begin{array}{c}\text { stimme eher } \\
\mathrm{zu} \\
2 \\
\end{array}$ & \multicolumn{2}{|c|}{$\begin{array}{c}\text { neutral } \\
3 \\
\end{array}$} & $\begin{array}{c}\text { stimme eher } \\
\text { nicht zu } \\
4 \\
\end{array}$ & \multicolumn{2}{|c|}{$\begin{array}{c}\text { stimme gar } \\
\text { nicht zu } \\
5 \\
\end{array}$} & \multirow{2}{*}{\begin{tabular}{|c|}
$\begin{array}{c}\text { keine } \\
\text { Beurteilung } \\
0\end{array}$ \\
$\Sigma$ \\
\end{tabular}} & \multirow{2}{*}{$\begin{array}{c}\text { Arithmetisches } \\
\text { Mittel }\end{array}$} & \multirow{2}{*}{\begin{tabular}{|c|}
$\begin{array}{c}\text { Standard- } \\
\text { abweichung }\end{array}$ \\
\pm \\
\end{tabular}} \\
\hline & & & & $\%$ & & & $\%$ & & & \\
\hline $\begin{array}{l}\text { In der Veranstaltung habe ich gelernt Podcasts zu } \\
\text { produzieren. }\end{array}$ & 70 & 20 & $\cdot$ & - & $1 x$ & - & - & $\cdot$ & 1,5 & 0,97 \\
\hline $\begin{array}{l}\text { Die praktischen Vorführungen und Übungen helfen mir } \\
\text { beim Verständnis der Lehrveranstaltung. }\end{array}$ & 78 & 11 & - & - & $\cdot$ & $1 x$ & 11 & - & 1,56 & 1,33 \\
\hline $\begin{array}{l}\text { Die Bedienung der Aufnahmetechnik (Audiorekorder, } \\
\text { Mikrofon) war eine besondere Herausforderung und } \\
\text { Hürde für mich. }\end{array}$ & 10 & $1 x$ & $6 x$ & 60 & - & $2 x$ & 20 & - & 3,1 & 1,2 \\
\hline $\begin{array}{l}\text { In ein Mikrofon zu sprechen, ber eitete mir zu Beginn } \\
\text { des Moduls Schwierigkeiten. }\end{array}$ & - & 30 & $3 x$ & 30 & 10 & $2 x$ & 20 & $1 x$ & 3,22 & 1,2 \\
\hline $\begin{array}{l}\text { In ein Mikrofon zu sprechen, bereitete mir zum Ende } \\
\text { des Moduls Schwierigkeiten. }\end{array}$ & & - & $2 x$ & 20 & 20 & $5 x$ & 50 & $1 x$ & 4,33 & 0,87 \\
\hline
\end{tabular}

Abbildung 3: Evaluation der Produktionsphase $(n=10)$. Quelle: eigene Darstellung

Im Anschluss an die Probeaufnahmen erfolgte die Aufnahme der Interviews mit den jeweiligen Gesprächspartnern. Einige Studierende haben sich dabei gewünscht, dass der Dozent am Gespräch teilnimmt und die Anmoderation sowie die Bedienung der Technik übernimmt.

\subsection{Post-Produktion - Schnitt und Audionachbearbeitung}

Für Schnitt und Audionachbearbeitung wurde im Projekt die Softwarelösung ultraschall.fm auf der Basis von reaper.fm eingesetzt. Aufgrund der Gestaltung der Lizenzen können Studierende hiermit ein Semester lang ohne entstehende Kosten arbeiten. Ultraschall.fm implementiert einen auf Podcasts zugeschnittenen Geschäftsprozess und bietet den Studierenden damit neben der softwaretechnischen Umsetzung auch die prozessorientierte Unterstützung für ein weitgehend eigenständiges Arbeiten. Zur Einleitung erhielten die Studierenden eine Einführung in das Themenfeld Post-Produktion mittels Software-Werkzeugen. Zur Erarbeitung der notwendigen Fertigkeiten in der Bedienung der Softwarelösungen haben die Studierenden die erstellten Probeaufnahmen verwenden können. Im Anschluss waren die Studierenden in der Lage, auch ihre eigenen Interviews mit den jeweiligen Gesprächspartnern in Schnitt und Audionachbearbeitung zu überführen. Der Dozent sowie eine studentische Hilfskraft standen hier nun im Wesentlichen noch unterstützend zur Seite.

Da die Studierenden keinerlei Vorwissen zum Themenkomplex Audio Engineering mit in die Veranstaltung bringen (dies ist nicht Bestandteil des Studiengangs Wirtschaftsinformatik B.Sc.), wurde im Anschluss an den Schnitt 
der Webdienst Auphonic ${ }^{4}$ zur weiteren Bearbeitung der Podcast-Episoden integriert. Auphonic ermöglicht die einfache Verbesserung der Tonqualität der Audiodateien und die Einbindung von Metadaten in die letztlich produzierten .mp3-Dateien. Darüber hinaus bietet Auphonic basale Audio EngineeringFunktionalitäten, die für den Umfang und die Ausrichtung des hier vorgestellten Podcast-Projekts passend sind (z.B. Audio Leveling, Loudness Normalization, Filtering, Noise and Hum Reduction).

\begin{tabular}{|c|c|c|c|c|c|c|c|c|}
\hline Kaffeepause Podcast-Projekt & $\begin{array}{c}\text { stimme voll } \\
\mathrm{zu} \\
1 \\
\end{array}$ & $\begin{array}{c}\text { stimme eher } \\
\mathrm{zu} \\
2 \\
\end{array}$ & $\begin{array}{c}\text { neutral } \\
3\end{array}$ & $\begin{array}{c}\text { stimme eher } \\
\text { nicht zu } \\
4 \\
\end{array}$ & $\begin{array}{c}\text { stimme gar } \\
\text { nicht zu } \\
5 \\
\end{array}$ & \begin{tabular}{|c|} 
keine \\
Beurteilung \\
0
\end{tabular} & $\begin{array}{c}\text { Arithmetisches } \\
\text { Mittel }\end{array}$ & \begin{tabular}{|l|} 
Standard- \\
abweichung
\end{tabular} \\
\hline $\begin{array}{l}\text { Ich habe mich bereits vor dem Modul mit Software zur } \\
\text { Audio-Nachbearbeitung beschäftigt. }\end{array}$ & - & $4 \times \quad 40$ & - & 10 & 50 & - & 3,7 & 1,49 \\
\hline \begin{tabular}{|l|} 
Die Bedienung der Software zur Audio- \\
Nachbearbeitung war eine besondere Herausforderung \\
und Hürde für mich.
\end{tabular} & - & - & $3 x$ & 11 & 56 & - & 4,22 & 0,97 \\
\hline
\end{tabular}

Abbildung 4: Evaluation der Produktionsphase $(n=10)$. Quelle: eigene Darstellung

\subsection{Publikation}

Auch zum Themenkomplex Publikation der erarbeiteten Ergebnisse erfolgte zunächst eine einleitende Vorstellung durch den Dozenten. Dabei wurden die gängigen Social-Media-Kanäle und Plattformen adressiert. Die produzierten Podcast-Episoden sind einer breiten Öffentlichkeit über die gängigen Plattformen (z.B. iTunes, panoptikum.io, podcast.de, Spotify) zugänglich. Technisch basiert dies auf der Generierung eines RSS-Feeds, der abonnierbar ist. Eine Verstetigung im Sinne einer studentischen Podcast-Reihe analog zu einem studentischen Online-Journal ist Bestandteil der Lehrinnovation. Die Podcast-Reihe wird auf einem selbstverwalteten Webdienst gehostet. Als Content-Management-System wird WordPress eingesetzt. Dieses ist den Studierenden des Moduls „Social Media“ bekannt und bedurfte keiner weiteren Einweisung. Zur Verwaltung der Media Assets wird das für WordPress und explizit für Podcaster entwickelte Plugin Podlove Podcast Publisher ${ }^{5}$ verwendet. Dieses bildet sämtliche Anforderungen ab, die für ein selbstgehostetes Podcast-Projekt notwendig sind. Podlove bietet darüber hinaus eine vollständige Integration des Auphonic-Webdienstes, welcher durch die Studierenden im Rahmen der Audionachbearbeitung verwendet wurde. Somit liegt auch hier der Fokus auf einer Vereinfachung der Prozessabläufe, so dass die Hürde des notwendigen Vorwissens für die Studierenden möglichst niedrig gehalten werden kann. Die Podcast-Episoden werden unter Creative Commons-Lizenz (CC BY-NC-ND 4.0 ${ }^{6}$ ) verbreitet. Zur Verbreitung werden die WordPress-Seite sowie die Einspeisung in die bekanntesten Podcast-Plattformen per RSSFeed genutzt. Begleitend wird im Modul die Ausarbeitung einer passgenauen Social-Media-Strategie thematisiert, auf die hier nicht weiter eingegangen werden soll.

4 https://auphonic.com (Zugriff am 19.08.2020).

5 https://publisher.podlove.org (Zugriff am 19.08.2020).

6 https://creativecommons.org/licenses/by-nc-nd/4.0/deed.de (Zugriff am 19.08.2020). 


\section{Abschließende Betrachtungen}

Begrüßenswert ist immer ein Austausch über Lehre und Lehrformate zwischen Dozenten. In festen Strukturen verankert, kann dies zu einer guten, gelebten Praxis zur fortlaufenden Verbesserung der Lehre beitragen. An der Hochschule Niederrhein ist aus der Hochschuldidaktik heraus die Initiative Le/Ni-Lehrforum hervorzuheben. Hier bieten sich Dozenten beste Möglichkeiten zu einem interdisziplinären Austausch über Lehre. In unkomplizierter Form können hier außerdem Workshops angeboten werden. Über diesen Weg konnten in der Folge der Umsetzung der hier vorgestellten Lehrinnovation bereits interessierte Interviewpartner für zukünftige Durchführungen des Projekts gewonnen werden. Darüber hinaus ist das Interesse geweckt worden, Podcasts für andere Lehrprojekte einzusetzen. Entsprechend ergeben sich hieraus Synergieeffekte: Die Durchführung von Podcast-Projekten könnten die Studierenden zukünftig auch als Dienstleistung für weitere Dozenten anbieten - und somit ihr Kompetenzportfolio als Social-Media-Manager vollumfänglich ausspielen. Hieraus sind erste Ideen zu einem lehrveranstaltungsübergreifenden Ausrollen der Lehrinnovation, z.B. zwischen den Bereichen Wirtschaftsinformatik und Marketing, aber auch mit der Chemie, einzelnen Instituten der Hochschule oder der Ökonometrie generiert worden. Neben einer fachbereichsinternen ergibt sich somit auch eine fachbereichsübergreifende Verwendbarkeit der Lehrinnovation. Gewünscht ist dabei insbesondere eine stärkere Vernetzung von Studierenden über verschiedene Studiengänge einer Hochschule hinweg. Letztlich wurde die Lehrinnovation in hochschulübergreifenden Barcamps zu digitaler Lehre vorgestellt. Ergänzend konnten Workshops im Rahmen von Konferenzen und Weiterbildungen für das wissenschaftliche Personal durchgeführt werden, in welche die Erkenntnisse aus der hier vorgestellten Lehrinnovation einflossen.

Und natürlich haben auch die Studierenden ein Feedback zu dieser Lehrinnovation gegeben: Besonders gefallen hat den Studierenden grundsätzlich die Produktion eines eigenen Podcasts als ein die Prüfungsphase überdauerndes Ergebnis. Dies ist in vielen Fällen auch mit einem gewissen Stolz auf das Ergebnis der Produktion verbunden. Die Beschäftigung mit dem Medium Podcast wurde als neu und interessant bewertet.

Auch Verbesserungspotentiale wurden identifiziert: So wurde die Interviewdauer auf 15 Minuten begrenzt. Hier hätten sich einige Studierenden mehr Freiheit (und Zeit) für eine freiere Ausgestaltung gewünscht. Auch mit Blick auf den ein oder anderen Versprecher wäre ein Wegfall des Zeitlimits hilfreich gewesen, weil einzelne Passagen im Zweifel mehrfach aufgenommen werden könnten. Die inhaltliche Fokussierung auf das Thema Abschlussarbeiten wurde in der mittelfristigen Sicht als zu sehr beschränkend wahrgenommen. Gerne hätten die Studierenden weitere Themen aus dem Hochschulalltag aufgegriffen oder wären intensiver auf die Geschichten hinter den Abschlussarbeiten eingegangen. Die Studierenden haben sich außerdem insbesondere eine Schulung zu Gesprächsaufbau, Gesprächstechnik und Rhetorik gewünscht. Die Einbindung der Podcast-Produktion als Prüfungsleistung hat 


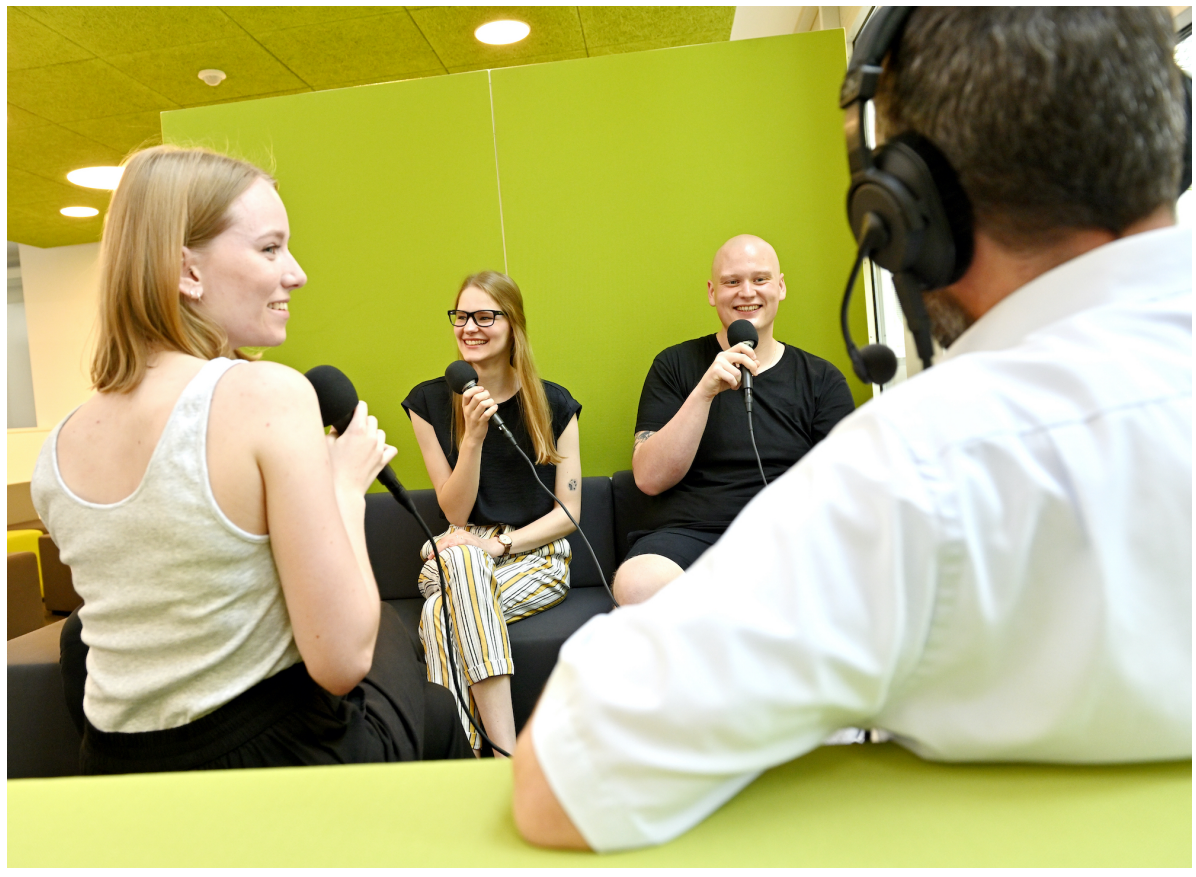

Abbildung 5: Aufnahmesituation. Quelle: Foto: Carlos Albuquerque / Hochschule Niederrhein

zu Unsicherheit bezüglich der Bewertungsmaßstäbe geführt. Und letztlich wurde gewünscht, noch mehr Übungsgespräche aufzuzeichnen, um mehr Routine zu bekommen.

In der kommenden Durchführung der Lehrveranstaltung soll diesen Kritikpunkten Rechnung getragen werden. Der Einsatz einer studentischen Hilfskraft, die bei den Aufzeichnungen der Interviews unterstützen wird, wird zu zeitlichen Freiräumen führen, welche zu einer höheren Flexibilität im Aufnahmezeitraster führen wird (Parallelisierung von Aufnahmezeitslots). Auch wird zukünftig der inhaltliche Fokus ausgeweitet und um allgemeine Themen aus dem Hochschulalltag ergänzt. Die inhaltliche Schwerpunktsetzung (Abschlussarbeiten, Hochschulalltag, Forschungsprojekte, studentisches Leben, ...) soll dabei im Titel der jeweiligen Episoden dargestellt werden, um den Hörern eine zügige Selektion der Inhalte zu ermöglichen. Entsprechend werden der Untertitel und das Logo des Podcasts auf Hochschulleben in 15 Minuten geändert. In zukünftigen Durchführungen wird außerdem eine Schulung zu Gesprächsaufbau, Gesprächstechnik und Rhetorik durch einen externen Referenten vorgesehen. Die Unsicherheit bezüglich der Bewertungsmaßstäbe soll durch die Bereitstellung einer Bewertungsmatrix und somit stärkerer Transparenz beantwortet werden.

Explizit wurde außerdem ermittelt, welche Maßnahmen geholfen haben, die Hürde des Sprechens in ein Mikrofon zu überwinden: Die Studierenden erläuterten hierzu, dass das Vormachen in Kleingruppen und bei lockerer Atmosphäre die wesentliche Hürde entfernt hat. Besonders bedeutsam waren dabei die Testaufnahmen und das damit verbundene Erproben der Best Practices sowie der Mikrofondisziplin. Dass der Dozent im Interview mit dabei war, wurde von den Studierenden als beruhigend wahrgenommen. 
Die Durchführung von Barcamps und Workshops durch den Dozenten hat zwischenzeitlich dazu geführt, dass weitere Podcast-Projekte innerhalb der Hochschule Niederrhein entstehen. Die Schwerpunkte liegen dabei ähnlich zu denen des Kaffeepause-Podcasts auf der Vorstellung studentischer Forschungsprojekte oder der Vorstellung der Professor:innen der Hochschule. Auch aus der Verwaltung werden Podcast-Angebote aufgebaut, z.B. für die Zentrale Studienberatung. Die Hochschule strebt daher an, die Podcasts auf einer zentralen Landing-Page gebündelt darzustellen. Die Kaffeepause ist also nur ein Beispiel für die erfolgreiche Einbindung eines Podcast in die Lehre und für die Verwendung in der Wissenschaftskommunikation. Gerade in Zeiten gebotener physischer Distanz und der Umstellung auf digitale Lehrund Lernformate bieten Podcasts reichhaltige Potentiale für Hochschulen.

\section{Literatur}

Copley, J. (2007). Audio and video podcasts of lectures for campus-based students: production and evaluation of student use. Innovations in Education and Teaching International, 44(4), 387-399. https://doi.org/10.1080/ 14703290701602805

Domenichini, B. (2018). Podcastnutzung in Deutschland. Media Perspektiven 2/2018, 46-49. Zugriff am 19.08.2020. Verfügbar unter: https://www.ardwerbung.de/fileadmin/user_upload/media-perspektiven/pdf/2018/0218_ Domenichini.pdf

Karla, J. (o.J.). Podcasts produzieren. jkarla.de. Zugriff am 19.08.2020. Verfügbar unter: http://www.jkarla.de/lehre/podcasts-produzieren/

Karla, J. \& Hoffmann, S. (2019). Podcasting in digitaler Lehre und Wissenschaftskommunikation. In T. Barton, C. Müller \& C. Seel (Hrsg.), Hochschulen in Zeiten der Digitalisierung (S. 173-182). Wiesbaden: Springer Vieweg. https://doi.org/10.1007/978-3-658-26618-9_11

Kay, R. H. (2012). Exploring the use of video podcasts in education: A comprehensive review of the literature. Computers in Human Behavior, 28(3), 820-831. https://doi.org/10.1016/j.chb.2012.01.011

Ketterl, M., Schmidt, T., Mertens, R. \& Morisse, K. (2006). Techniken und Einsatzszenarien für Podcasts in der universitären Lehre. In Proceedings der PreConference Workshops der 4. e-Learning Fachtagung der GI (S. 81-90). Zugriff am 19.08.2020. Verfügbar unter: http://www2.informatik.uni-osnabrueck.de/ papers_pdf/2006_05.pdf

Kirkwood, A. \& Price, L. (2014). Technology-enhanced learning and teaching in higher education: what is 'enhanced' and how do we know? A critical literature review. Learning, Media and Technology, 39(1), 6-36. https://doi.org/10. 1080/17439884.2013.770404 
Kronen, M. \& Karla, J. (2007a). Problemfelder des Einsatzes von Podcasting im unternehmensinternen Wissensmanagement. In K. Meißner \& M. Engelien (Hrsg.), Virtuelle Organisation und Neue Medien 2007 (S. 387-402). Dresden: TUDpress. Zugriff am 19.08.2020. Verfügbar unter: https://d-nb.info/1068446234/34

Kronen, M. \& Karla, J. (2007b). Podcasting as a Tool in Organisational Knowledge Management. In P. Kommers (Hrsg.), e-Society 2007 (S. 220-228). Lissabon: IADIS Press.

McGarr, O. (2009). A review of podcasting in higher education: Its influence on the traditional lecture. Australasian Journal of Educational Technology, 25(3), 309-321. https://doi.org/10.14742/ajet.1136.

McKinney, D. Dyck, J. L. \& Luber, E. S. (2009). iTunes University and the classroom: Can podcasts replace Professors? Computers \& Education, 52(3), 617-623. https://doi.org/10.1016/j.compedu.2008.11.004. 